\title{
Functional and systemic effects of whole body electrical stimulation post bariatric surgery: study protocol for a randomized controlled trial
}

Larissa Delgado André ${ }^{1}$, Renata P. Basso-Vanelli ${ }^{1}$, Luciana Di Thommazo-Luporini ${ }^{1}$, Paula Angélica Ricci ${ }^{1}$, Ramona Cabiddu' ${ }^{1}$, Soraia Pilon Jürgensen ${ }^{1}$, Claudio Ricardo de Oliveira ${ }^{2}$, Ross Arena ${ }^{3}$ and Audrey Borghi-Silva ${ }^{4^{*}}$ (DD

\begin{abstract}
Background: Obesity represents a major public health problem and is the fifth leading risk factor for mortality. Morbid obesity is associated with chronic systemic inflammation which increases the risk of comorbidities. Bariatric surgery (BS) is considered an effective intervention for obese patients. However, BS is associated with dietary restriction, potentially limiting physical activity. Whole-body neuromuscular electrical stimulation (WBS) could represent an innovative option for the rehabilitation of BS patients, especially during the early postoperative phase when other conventional techniques are contraindicated. WBS is a safe and effective tool to combat sarcopenia and metabolic risk as well as increasing muscle mass, producing greater glucose uptake, and reducing the proinflammatory state. Therefore, the objective of this study is to evaluate the effects of WBS on body composition, functional capacity, muscle strength and endurance, insulin resistance, and pro- and anti-inflammatory circulating markers in obese patients undergoing BS.
\end{abstract}

Methods/design: The present study is a randomized, double-blind, placebo-controlled, parallel groups clinical trial approved by the Ethics Committee of our Institution. Thirty-six volunteers (body mass index (BMI) $>35 \mathrm{~kg} / \mathrm{m}^{2}$ ) between 18 and 45 years of age will be randomized to the WBS group (WBSG) or control (Sham) group (ShamG) after being submitted to BS. Preoperative assessments will include maximal and submaximal exercise testing, body composition, blood inflammatory markers, and quadriceps strength and endurance. The second day after discharge, body composition will be evaluated and a 6-min walk test (6MWT) will be performed. The WBS or Sham protocol will consist of 30 daily sessions for 6 consecutive weeks. Afterwards, the same assessments that were performed in the preoperative period will be repeated.

Discussion: Considering the important role of WBS in skeletal muscle conditioning and its value as an aid in exercise performance, the proposed study will investigate this technique as a tool to promote early rehabilitation in these patients, and as a strategy to enhance exercise capacity, weight loss, and peripheral muscle strength with positive systemic effects. The present study is still ongoing, and data will be published after its conclusion.

Trial registration: REBEC, RBR-99qw5h. Registered on 20 February 2015.

Keywords: Electrical stimulation, Obesity, Functional capacity, Systemic markers, Surgery

* Correspondence: audrey@ufscar.br

${ }^{4}$ Cardiopulmonary Physiotherapy Laboratory, Department of Physiotherapy,

Federal University of São Carlos, Rod. Washington Luís, km 235, São Carlos,

São Paulo 13565-905, Brazil

Full list of author information is available at the end of the article

(c) The Author(s). 2018 Open Access This article is distributed under the terms of the Creative Commons Attribution 4.0 International License (http://creativecommons.org/licenses/by/4.0/), which permits unrestricted use, distribution, and reproduction in any medium, provided you give appropriate credit to the original author(s) and the source, provide a link to the Creative Commons license, and indicate if changes were made. The Creative Commons Public Domain Dedication waiver (http://creativecommons.org/publicdomain/zero/1.0/) applies to the data made available in this article, unless otherwise stated. 


\section{Background}

Obesity is a chronic disease that has reached epidemic proportions in children and adults; it is currently the fifth leading risk factor for mortality [1]. According to the World Health Organization (WHO), obesity has been growing at an alarming rate in the last decade and is becoming a significant public health problem $[2,3]$. Obesity is a complex multifactorial disorder associated with derangements in immune function, low-grade inflammation, and changes in the amount of pro- and anti-inflammatory cytokines [4]. Adipose tissue is a metabolically dynamic, endocrine organ that participates in the regulation of physiological processes, including immunity-related processes, through the release of proand anti-inflammatory cytokines [4]. These include leptin, myostatin, adiponectin, and tumor necrosis factor (TNF)- $\alpha$, all of which are involved in the development of cardiometabolic diseases, such as type II diabetes, and cardiovascular disease (CVD) [4].

Adipose tissue is recognized as an active tissue able to influence food intake control, energy balance, insulin action, lipids, glucose metabolism, angiogenesis, vascular remodeling, immunity, and inflammation [3, 4]. Obesity also leads to musculoskeletal dysfunction and reduces cardiorespiratory fitness as well as muscle strength, endurance, and flexibility. These effects often significantly contribute to chronic pain and functional limitations [5].

Many patients do not respond effectively to conservative therapeutic interventions for weight reduction and require a surgical approach, which is considered safe with a low mortality rate [6-8].

Conventional gastric bypass surgery, associated with dietary restriction in the postoperative (PO) period, limits physical activity performance and can lead to reductions in skeletal muscle mass and performance [9]. As such, strategies are needed to preserve and ideally enhance functional performance. In this context, whole-body neuromuscular electrical stimulation (WBS), which differs from conventional electrical stimulation, has been recently introduced as a strategy to simultaneously stimulate several large muscle groups. It represents a promising and innovative option to enhance weight loss, improve exercise capacity and peripheral muscle strength, and induce positive systemic effects [10-12]. Several studies have demonstrated that WBS reduces sarcopenia and abdominal fat mass in both young and older sedentary individuals [13]. These studies emphasize the potential effects of WBS-being able to act beneficially when other conventional exercises are contraindicated [13].

Another study showed that WBS could, through an increase in muscle mass, potentiate the action of insulin and thus produce greater uptake of glucose in the muscle as well as reduce the proinflammatory state. These findings may be particularly relevant in the obese population which is predisposed to greater insulin resistance [14].

While WBS may hold promise as a rehabilitation strategy following bariatric surgery (BS), especially in the early stages after surgery, there appears to be no evidence about its applicability in the bariatric PO period.

Therefore, the objective of this study is to evaluate the effects of WBS on body composition, functional capacity, muscle strength and endurance, insulin resistance, and pro- and anti-inflammatory circulating markers in obese patients undergoing BS in the early PO period.

We hypothesize that WBS would enhance functional capacity, muscle strength, and endurance, and reduce inflammation in BS patients when compared with controls (Sham). Moreover, we hypothesized that WBS would reduce fat mass, improve muscle mass, and positively affect insulin resistance and exercise capacity in these patients. We also expect patients to present good acceptance and adherence to the intervention protocol.

\section{Methods/design Study design}

The present study is a randomized, double-blind, placebo-controlled, parallel groups clinical trial conducted at the Cardiopulmonary Physical Therapy Laboratory (LACAP) of the Federal University of São Carlos (UFSCar), São Carlos, SP, Brazil. This study was approved by the UFSCar Ethics Committee (number 966.613) and registered at the Brazilian Registry of Clinical Trials (ReBEC, RBR-99qw5h) on 20 February 2015. Recruited volunteers will all provide written informed consent prior to participation. The study will respect the Consolidated Standards of Reporting Trials (CONSORT) as show in the Fig. 1 with the template of content for the schedule of enrolment, interventions, and assessments. The study will also respect the Standard Protocol Items: Recommendations for Interventional Trials (SPIRIT) as shown in Additional file 1 , respectively.

\section{Volunteers \\ Recruitment, randomization, and blinding}

Volunteers will be recruited from among patients included in a waiting list for BS and followed by a multidisciplinary team (composed of a physician, surgeon, anesthesiologist, cardiologist, physical therapist, nutritionist, and psychologist) for a minimum period of 1 year. During this period, volunteers will be counseled on smoking and alcohol cessation and informed about the surgical procedure and PO care.

Eligible volunteers will be evaluated within 1 month before BS, 2 days after discharge and, afterwards, will be randomized to the experimental or control group with a 1:1 allocation ratio by a computer-based permuted block randomization (www.randomization.com). To achieve a 


\begin{tabular}{|c|c|c|c|c|c|c|}
\hline & \multirow{3}{*}{$\begin{array}{c}\begin{array}{c}\text { Enrolmen } \\
\mathbf{t}\end{array} \\
\mathrm{TO} \\
\end{array}$} & \multicolumn{5}{|c|}{ STUDY PERIOD } \\
\hline & & \multirow{2}{*}{$\begin{array}{c}\begin{array}{c}\text { Pre } \\
\text { allocation }\end{array} \\
\mathbf{T 1} \\
\end{array}$} & \multirow{2}{*}{$\begin{array}{c}\text { Allocation } \\
T 2\end{array}$} & \multicolumn{3}{|c|}{ Post-allocation } \\
\hline TIMEPOINT & & & & $T 3$ & Intervention & $T 4$ \\
\hline \multicolumn{7}{|l|}{ ENROLMENT: } \\
\hline \multirow{3}{*}{$\begin{array}{r}\text { Eligibility screen } \\
\text { Written informed } \\
\text { consent } \\
\text { Allocation }\end{array}$} & $\mathrm{X}$ & & & & & \\
\hline & $\mathrm{X}$ & & & & & \\
\hline & & & $\mathrm{X}$ & & & \\
\hline \multicolumn{7}{|l|}{$\begin{array}{r}\text { INTERVENTION } \\
\text { S: }\end{array}$} \\
\hline \multirow{2}{*}{$\begin{array}{r}\text { [EENMC Group] } \\
\text { [Sham Group] }\end{array}$} & & & & & $\mathrm{X}$ & \\
\hline & & & & & $\mathrm{X}$ & \\
\hline \multicolumn{7}{|l|}{ ASSESSMENTS: } \\
\hline \multicolumn{7}{|l|}{$\begin{array}{r}\text { [Anamnesis and } \\
\text { Baecke } \\
\text { questionnaire] }\end{array}$} \\
\hline \multirow{4}{*}{$\begin{array}{r}{[\mathrm{CPX}]} \\
\\
{[6 \mathrm{MWT}]} \\
\text { [Bioimpedance } \\
\text { measurement] }\end{array}$} & & $\mathrm{X}$ & & & & $\mathrm{X}$ \\
\hline & & $\mathrm{X}$ & & $\mathrm{X}$ & & $\mathrm{X}$ \\
\hline & & $\mathrm{X}$ & & $\mathrm{X}$ & & $\mathrm{X}$ \\
\hline & & $\mathrm{X}$ & & & & \\
\hline $\begin{array}{r}\text { [Blood collection] } \\
\text { [Isokinetic, } \\
\text { isometric strength } \\
\text { and endurance } \\
\text { assessments] }\end{array}$ & & $\mathrm{X}$ & & & & $\mathrm{X}$ \\
\hline
\end{tabular}

Fig. 1 Template of content for the schedule of enrolment, interventions, and assessments. T0: baseline variables; T1: preoperative evaluations; T2: allocation; T3: postoperative evaluation; T4: postintervention evaluation. 6MWT 6-min walking test, CPX symptom-limited or maximum cardiopulmonary exercise testing, WBS (Whole-body neuromuscular electrical stimulation)

gender balance for both protocols, the randomization will be stratified by gender and two randomization plans for each gender will be proposed with blocks of 4 . The randomization sequence will be generated by an independent research assistant and the intervention protocol will be applied by a physical therapist. Since the present study is a double-blind clinical trial, the volunteers, the physical therapist who will perform the assessments, and the researcher who will perform the statistical analysis will be blinded to the allocation of the volunteers.

\section{Inclusion and exclusion criteria}

Inclusion criteria will be as follows: 1) body mass index (BMI) in class I $\left(30-34.9 \mathrm{~kg} / \mathrm{m}^{2}\right)$, class II (BMI 35$\left.39.9 \mathrm{~kg} / \mathrm{m}^{2}\right)$, or class III (BMI $\geq 40 \mathrm{~kg} / \mathrm{m}^{2}$ ) obesity range for at least 3 years or more [15]. Patients with a BMI in the $30-34.9 \mathrm{~kg} / \mathrm{m}^{2}$ range and presenting with associated comorbidities are candidates for BS at the discretion of medical staff and the endocrinologist [15]. Therefore, these patients will also be included in the present study);
2) no response to conventional and conservative treatment (pharmacological or not); 3) aged between 18 and 45 years; and 4) candidates for gastric bypass surgery.

Exclusion criteria will be as follows: 1) orthopedic conditions (arthrosis or pain) or neurological impairments that would preclude physical activity; 2) myocardial infarction within the last 6 months before study initiation; 3) implanted pacemaker or any metallic device; 4) unstable angina; 5) chronic heart rhythm disorders; 6) moderate or severe heart valve disease; 7) consistent history of heart disease; 8) uncontrolled hypertension; 9) uncontrolled and/or insulin-dependent diabetes mellitus; 10) $\beta$-blocker use; 11) chronic obstructive pulmonary disease (COPD) or other lung disorders; 12) any contraindication to the cardiopulmonary exercise test (CPX); 13) any health issues that compromise functional capacity testing; 14) distal arteriopathies; 15) any inflammatory, kidney, or liver conditions with documented diabetic neuropathy; 16) cognitive impairments or difficulty in comprehending or adhering to the procedures; 17) smoking; 18) use of drugs; 19) gestation; 20) use of psychotropic medications, 
anxiolytics, antidepressants, or appetite control medications; 21) postmenopausal women; and 22) concurrent participation in a regular exercise program. Patients with PO complications will also be excluded from this study.

\section{Sample size}

The sample size calculation was performed using GPower, Version 3.1.3 (Franz Faul Universität Kiel, Germany), based on a previous study by our group performed on BS patients [16] which showed a difference between the distance walked during a 6-min walking test (6MWT) before and after aerobic training of $49.7 \pm$ $15 \mathrm{~m}$. We hypothesize that we will see an improvement in $6 \mathrm{MWT}$ distance following WBS to a similar level as our previous study. Therefore, we projected a sample size that would provide $80 \%$ power at a significance level of $\alpha=0.05$, detecting a variation of $30 \%$ as a favorable outcome. These parameters indicate a sample of five volunteers would be required to investigate functional gains after the intervention.

The primary outcome measure for the study will be the 6MWT distance in meters since the distance walked following interventions in patients with chronic conditions is an important tool to assess the efficacy of interventions that theoretically improve functional capacity [16]. Secondary outcomes of this study are to investigate whether the intervention prevents muscle mass loss and whether there is an association between 6MWT distance with lean mass in kilograms [17]. Therefore, since the performance improvement, the prevention of lean mass loss, and the association between these measures represent important outcomes in the present study, the sample size was recalculated. Based on the bivariate normal correlation model, considering a significance level of $5 \%$, a sample power of $95 \%$, and a strong correlation $(>0.70)$ between lean mass percentage and 6MWT performance as the main outcome, the calculated sample size was 16 volunteers per group. Anticipating a dropout rate of approximately $15 \%$, a total of 36 patients will be included in the present study.

\section{Procedures}

Before applying the intervention protocol, volunteers will be familiarized with assessment procedures and the WBS technique. Volunteers will be evaluated in the afternoon to avoid different physiological responses due to circadian changes; they will be instructed not to drink caffeine, alcohol, or any other stimulant the night before as well as on the day of data collection, and not to perform strenuous exercises the day before data collection. All experiments will be carried out in an acclimatized room, with a controlled temperature between $22{ }^{\circ} \mathrm{C}$ and $24{ }^{\circ} \mathrm{C}\left(71.6{ }^{\circ} \mathrm{F}\right.$ to $\left.75.2^{\circ} \mathrm{F}\right)$ and relative air humidity between $40 \%$ and $60 \%$.

\section{Evaluations (testing procedures or measurements)}

All enrolled volunteers will be submitted to anamnesis and the Baecke questionnaire will be administered to collect information about habitual physical activity related to occupation, sports, and leisure activities [18].

After the screening period and signing the informed consent (Additional file 2), the volunteers will return to the laboratory on 4 different days to perform the preoperative evaluations (Fig. 2), which are: 1) maximal CPX; 2) the 6MWT; 3) blood collection; 4) bioimpedance measurements; and 5) isokinetic and isometric peripheral muscle strength and endurance assessments. All evaluations will be performed again following the intervention.

The CPX will be performed on a treadmill (Super ATL, Porto Alegre, Rio Grande do Sul, Brazil) using the incremental Bruce protocol. Volunteers will be monitored by a 12-lead electrocardiogram (WinCardio System, Microme, Brasilia, Brazil) supervised by a physician, and encouraged to achieve maximal effort. Heart rate, blood pressure, and subjective responses of dyspnea and fatigue by the CR10 scale [19] will also be measured.

The 6MWT will be performed according to guidelines established by the ATS/American College of Chest Physicians [20]. The object of this test is to walk as far as possible for $6 \mathrm{~min}$ over a 30-m corridor. Cardiorespiratory and metabolic variables will be collected breath-by-breath through a portable Oxycon Mobile ergospirometer (Mijnhardt/Jäger, Würzburg, Germany) throughout the 6MWT.

Blood samples will be collected the morning after a 12-h fast from the upper limb antecubital vein by a qualified professional to quantify fasting glucose levels, insulin, the insulin resistance index by the Homeostasis Model Assessment method (HOMA-IR), and the lipid profile (total cholesterol, high-density lipoprotein cholesterol (HDL-C), low-density lipoprotein cholesterol (LDL-C), very low-density lipoprotein cholesterol (VLDL-C), and triglycerides) [2, 21]. It will also be used to measure the serums concentrations of TNF- $\alpha$, adiponectin, leptin, and myostatin. These samples will be analyzed by enzyme-linked immunosorbent assay (ELISA) in duplicates using high-sensitivity kits (Quantikine ${ }^{\circ}$ HS, R\&D Systems, Minneapolis, USA) according to the manufacturer's recommendations [22]. The volunteers will be instructed not to exercise for $48 \mathrm{~h}$ preceding the examination and to maintain their usual diet.

Body composition will be obtained by bioelectrical impedance analysis, performed by an InBody 720 device and dual energy $\mathrm{x}$-ray technique using digital absorptiometry (DXA) [23-26]. The use of both equipment for body composition will ensure all data is collected since the DXA scanning area can be limited and compromise some measures due to the larger abdominal circumference of recruited obese patients. Volunteers will be instructed to undergo a 4-h fast, to wear light clothes, not to have any 


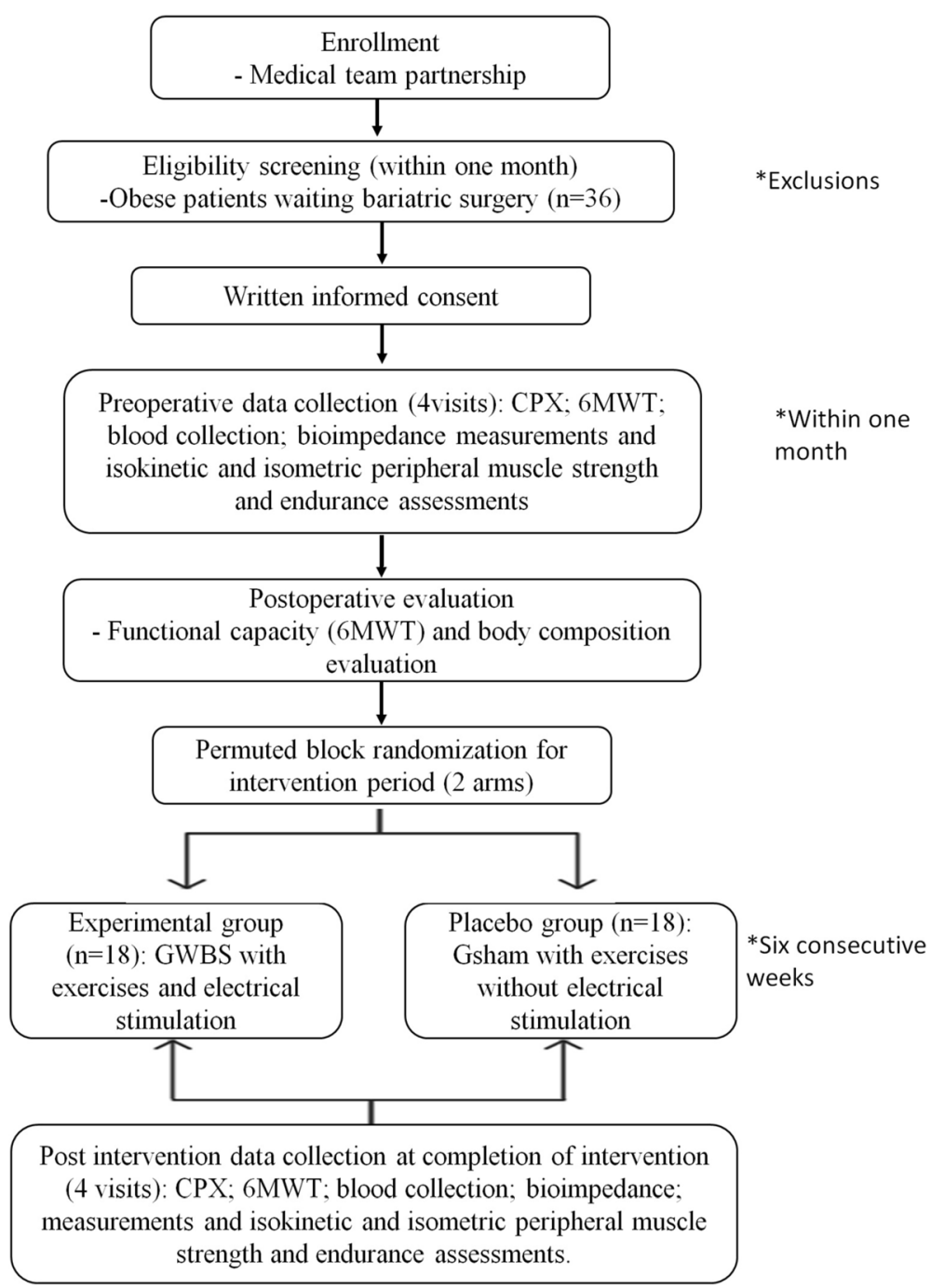

Fig. 2 Consolidated Standards of Reporting Trials (CONSORT) flow diagram of the study. 6MWT 6-min walking test, CPX symptom-limited or maximum cardiopulmonary exercise testing

metal objects in contact with their body, to urinate prior to the examination, not to drink alcohol, and not to perform strenuous exercises the day before the examination [23].

Evaluations of concentric and isometric extension of the dominant knee will be carried out using an isokinetic dynamometer (Biodex Multi-Joint System 3; Biodex Medical System, Inc., Shirley, NY) at least $48 \mathrm{~h}$ after the last functional test.

Isometric and isokinetic assessments will be performed using a previously described protocol [27]. The highest absolute concentric extensor and flexor torque as well as a BMI-normalized value, the flexor/extensor ratio, total work, power, and quadriceps femoris muscle fatigue at an angular speed of $60^{\circ} \mathrm{s}$ will be measured [27, 28]. Volunteer positioning and the alignment of the mechanical axis, as well as the gravitational correction, will be performed as previously described [27]. Standardized encouragement will be provided by a single evaluator to stimulate volunteers to produce their maximal effort during all tests.

\section{Bariatric surgery}

Many patients do not respond effectively to conservative therapeutic interventions and require a surgical approach. In recent years, the Roux-en- $Y$ gastric bypass technique (RYGB) has been the most predominant modality of surgery, and is proven to be safe, leading to low mortality $[6,8]$. It combines gastric restriction and reconstruction of intestinal transit through a jejunal loop that looks like the letter Y [8]. RYGB can provide greater weight loss in the long term with a reduction in several risk factors for comorbidities $[8,9]$. 


\section{Postoperative data collection}

In the PO period, the $6 \mathrm{MWT}$ and body composition measurements will be performed. These evaluations will occur within 1 week after discharge. Afterwards, volunteers will be randomized to the experimental group or the control group. At the end of the interventional trial, all preoperative evaluations will be performed a second time.

\section{Intervention protocol (WBS or sham protocol)}

Following the PO assessments and subsequent randomization, the volunteers will return to the laboratory to perform the intervention protocol with WBS equipment (Miha Bodytec, Augsburg, Germany) [29] as described in other studies [30-32]. The experimental group (WBSG; $n=16$ ) will perform the WBS protocol while the control group (ShamG; $n=16$ ) will perform the same protocol without WBS stimulus.

The WBS vests and cuff electrodes allow for simultaneous control and innervation of 14-18 muscle groups or 10 regions (upper legs, upper arms, bottom, abdomen, chest, lower back, upper back, including the latissimus dorsi) with a selectable intensity for each one allowing a total electrode area of up to $2800 \mathrm{~cm}^{2}$ [32, 33]. The strain and current intensity can be individually selected and modified during the protocol and be progressively increased during each training session. The parameters will be saved on smart cards to ensure reliable and valid application during the WBS protocol [31, 32].

WBS stimulation will be accompanied by low-intensity movements to allow an effective contraction (maximum voluntary contraction requested by the supervisor) during electrical stimulation. The exercises will be carried out without any additional weights with volunteers in the standing position. Specifically, WBS will be performed in conjunction with 10-14 dynamics: squat, trunk flexion, exercises of upper limbs, and isometric abdomen contraction. In the experimental group, these muscle contractions will be performed simultaneously with an electrical stimulation current. Figure 3 illustrates a volunteer performing the protocol with a WBS vest.

The sessions will last approximately $20-30$ min using a previously described protocol, involving a rectangular wave by a symmetrical bipolar electric pulse [13]. Considering a total of 6 weeks of training for both groups, the WBS protocol will be performed in three nonconsecutive days of endurance alternating with 2 days of training per week. All sessions will occur with the supervision of a trained physical therapist.

The endurance training session will apply a bipolar electrical current with a frequency of $85 \mathrm{~Hz}$ and an impulse breadth of $350 \mu$ s intermittently with $6 \mathrm{~s}$ of electrical stimulation using a direct impulse boost to perform the exercise and $4 \mathrm{~s}$ off for rest, with ramp of $0.4 \mathrm{~s}$. The total time under current per session will be approximately $12 \mathrm{~min}$, with approximately $5 \mathrm{~min}$ of rest between sets. The strength sessions will consist of the same bipolar electrical current, with a frequency of $30 \mathrm{~Hz}$ and impulse breadth of $350 \mu \mathrm{s}$ intermittently, $4 \mathrm{~s}$ of electrical stimulation and $10 \mathrm{~s}$ of rest, with ramp of $0.4 \mathrm{~s}$. These sessions will last approximately $8 \mathrm{~min}$, with approximately $5 \mathrm{~min}$ of resting between exercises.

The intensity of both sessions is prescribed to reach submaximal levels, which will be adjusted to each region according to disparities of current sensitivity, respecting visual and effective contraction, without volunteer discomfort [30,31]. To maintain an adequate and tolerable exercise intensity, the BORG scale was used. Thus, volunteers should score between 2 and 3 for the perception of effort in this scale in the endurance protocol and above 5 in the protocol of force [31,32].

The difference between the WBS and control intervention is that the intensity of electrical stimulation will be switched off in the latter group to avoid any influence of WBS equipment during the exercises. Volunteers will be instructed not to perform any regular exercise activities, being questioned weekly with respect to compliance with these recommendations. After the postintervention assessment, the WBS protocol will be offered to the control group.

\section{Postintervention data collection}

All preoperative assessments will be performed at the end of the intervention period. At the end of the protocol and assessments, all volunteers will receive a report with all data evaluations.

\section{Statistical analysis}

A blinded researcher will perform all statistical analyses for this study. The volunteers will be codified by the researcher responsible for randomization to ensure blinding of control and intervention groups. Descriptive statistics including the mean, standard deviation (SD), and quartiles will be applied to summarize the data. The Shapiro Wilk test will be used to investigate data distribution. Data will be expressed as mean, SD, and 95\% confidence interval. The primary analysis is to assess the effect of the intervention on 6MWT distance and the secondary analysis is the relationship between 6MWT distance and the lean mass loss.

In this sense, the effect of WBS will be assessed in the context of the following hypotheses:

H0: WBS does not improve 6MWT distance and/or does not prevent the loss of muscle mass.

H1: WBS increases 6MWT distance and/or prevents the loss of muscle mass.

If the data present a normal distribution, analysis of variance (ANOVA) will be tested for comparisons 


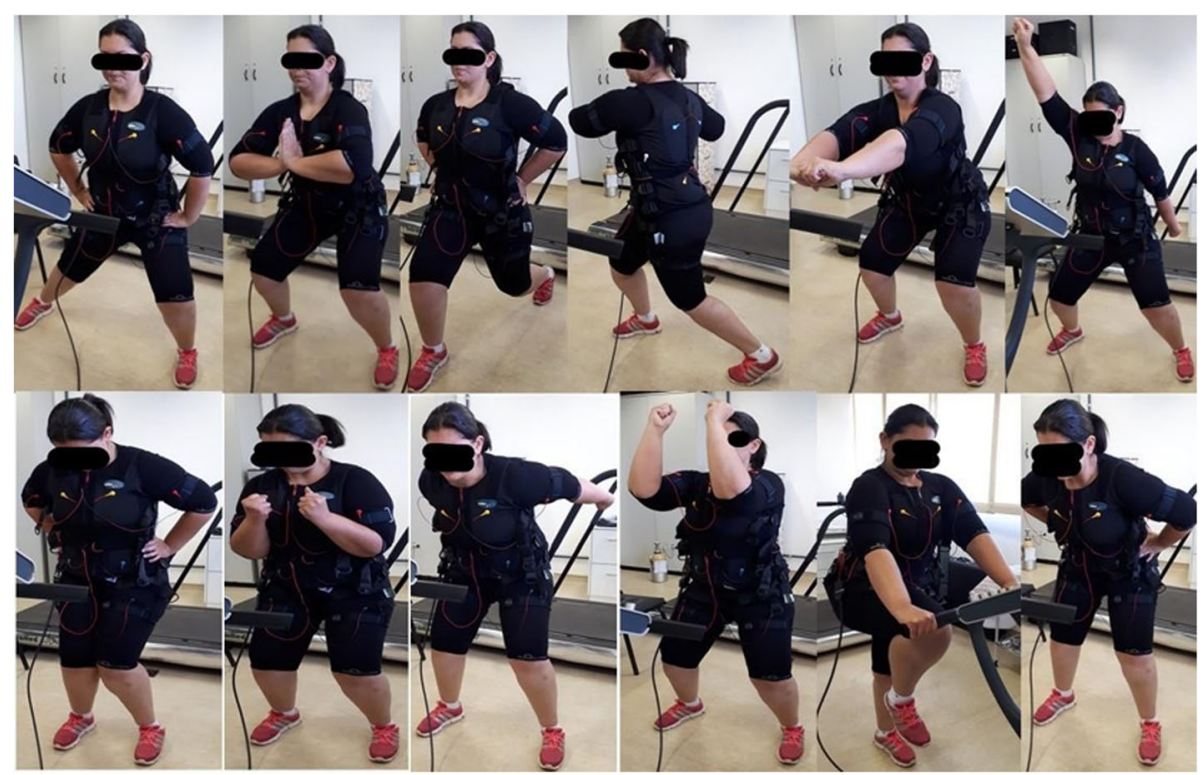

Fig. 3 Whole-body electrical stimulation exercises performed by a recruited volunteer

between the body composition and 6MWT distance change considering three periods of evaluation (preoperative, postoperative, and postintervention). In instances where data are not normally distributed, nonparametric tests will be applied.

The benefits obtained by both groups will be demonstrated through the difference between postintervention results minus PO data with all variables that will be collected. Comparisons between groups will be assessed using the unpaired Student $t$ test or Mann-Whitney test, as appropriate.

Effect size will be analyzed using Cohen's $d$, and the results will be interpreted based on the Cohen classification [34] as follows: small (0.21-0.49), medium $(0.50-0.79)$, or large (>0.80).

The statistical analysis using correlation will assess the degree of association (or dependence) between improvements in functional tests, body composition data (InBody 720 and DXA), muscular assessment (dynamometry), and concentration of cytokines.

Appropriate correlation tests will be used if normality of distribution, linearity, variability, and homoscedasticity is satisfied from the scatter diagram performed a priori. The correlations will be defined as weak $(0.20-$ $0.50)$, strong $(0.50-0.70)$, and very strong $(>0.70)$ [35]. The statistical analysis will be performed using SPSS Statistics for Windows, Version 17.0. (SPSS Inc., Chicago, IL, USA) and MedCalc, version 11.4.4.0 (MedCalc Software, Mariakerke, Belgium), adopting a significance level of 5\%. Per-protocol and intention-to-treat analyses will be conducted.

\section{Discussion}

Among bariatric surgical techniques, the RYGB has brought satisfactory long-term results such as increased weight loss and subsequent maintenance, with a concomitant reduction in several risk factors in bariatric patients. In this sense, previous investigations have demonstrated it is a more effective procedure compared with other surgical approaches [36, 37]. Some longitudinal studies observed that different isolated bariatric surgical techniques have a positive influence on reducing weight at 6 and 12 months, improving functional capacity, and even reducing comorbidities associated with obesity [38-40]. However, it has recently been demonstrated that the inclusion of structured physical activity in the short term (within 4 months of the surgical procedure) can also result in functional capacity improvement [41, 42].

On the other hand, most studies in which physical exercise have been proposed after BS results in considerable attrition with low adherence to exercise rehabilitation and weight-loss programs [15, 43-46]. Another important feature is the severe dietary restriction especially in the first 3 months after surgery that is recommended during the early PO period [9]. This stage involves a reduction in protein intake which affects fat but also lean mass leading to accelerated weight loss [47]. In the early PO period, patients submitted to gastric bypass surgery should start a liquid diet on the first day and maintain this diet for the first week following surgery [48]. Associated with this, patients have a surgical rest period with a restriction on moderate to high-intensity exercise [49]; pain is also a frequent issue following surgery. Thus, promoting physical 
activity in this patient population following surgery is a challenge [9].

In this context, intervention strategies achievable as soon as possible after the surgical procedure could have a positive impact in this population. Current evidence has shown that WBS may be an alternative and potentially beneficial approach associated with conventional physical exercise programs in different populations [12].

A contemporary study demonstrated that WBS positively effects sarcopenia and abdominal body fat parameters across the lifespan and in sedentary volunteers [13]. This research highlights the potential effects of this electromyostimulation technique and its beneficial impact on body composition and functional capacity which may overcome some limitations of conventional physical exercise programs, especially when it is contraindicated [13]. Another recent study indicated that a WBS program was feasible and effective for elderly volunteers, being a favorable alternative for improving body composition and physical strength in postmenopausal and overweight women $[11,33]$.

Furthermore, positive effects of WBS on muscle parameters have been previously observed in athletes [10], healthy young individuals [49], the elderly, and patients with chronic disease [50, 14]. Additionally, other studies have shown that WBS could increase muscle mass, enhance insulin action and, thus, induce a greater glucose uptake into skeletal muscle [51]. These findings may be of particular relevance, especially in obese individuals who are predisposed to higher levels of insulin resistance [14]. A recent study found that WBS application to the abdomen for 12 weeks was effective in reducing a proinflammatory state (as observed by TNF- $\alpha$, adiponectin, and C-reactive protein levels) [51], as well as fasting glycemia, insulin resistance, and visceral adiposity in obese diabetics [5]. However, the potential effects of this technology on inflammatory markers after BS are unknown and warrant further study.

Additionally, it is important to emphasize that WBS equipment can penetrate subcutaneous tissue and stimulate deeper muscles due to the ability to select high current intensities. Thus, WBS can be considered an innovative and promising technique in obese individuals who have difficulty performing exercise, emphasizing the importance of this study.

Data will be published after completion of the study.

\section{Trial status}

Patient recruitment is currently underway.

\section{Additional files}

Additional file 1: SPIRIT 2013 checklist: recommended items to address in a clinical trial protocol and related documents. (DOC $122 \mathrm{~kb}$ )

Additional file 2: Model consent form. (DOCX $16 \mathrm{~kb}$ )

\section{Abbreviations}

6MWT: Six-minute walking test; BMI: Body mass index; BS: Bariatric surgery; COPD: Chronic obstructive pulmonary disease; CPX: Cardiopulmonary exercise test; CVD: Cardiovascular disease; DXA: Dual energy $\mathrm{x}$-ray technique using digital absorptiometry; ELISA: Enzyme-linked immunosorbent assay; HDL-C: High-density lipoprotein cholesterol; LDL-C: Low-density lipoprotein cholesterol; PO: Postoperative; RYGB: Roux-en-Y gastric bypass technique; ShamG: Control group; TNF: Tumor necrosis factor; VLDL-C: Very low-density lipoprotein cholesterol; WBS: Whole-body neuromuscular electrical stimulation; WBSG: Whole-body neuromuscular electrical stimulation group; WHO: World Health Organization

\section{Funding}

This study has the financial support of São Paulo State Research Foundation (FAPESP; grant 2015/04101-1).

\section{Authors' contributions}

LDA contributed to the conception and experimental design and manuscript writing and will contribute to data collection. RPBV contributed to the conception and experimental design, manuscript writing and analysis. PAR, SPJ, and CRdO will contribute to data analysis and collection and contributed by revising the manuscript. LDTL, RC, and RA contributed to manuscript writing and final approval of the version to be published. ABS contributed to the conception and experimental design, manuscript revision/editing, and final approval of the version to be published, and will supervise its execution, data analysis, and interpretation. All authors read and approved the final manuscript.

Ethics approval and consent to participate

All study protocols were reviewed and approved by the UFSCar Ethics Committee (number 966.613). Patient informed consent will be obtained.

Consent for publication

Not applicable.

\section{Competing interests}

The authors declare that they have no competing interests.

\section{Publisher's Note}

Springer Nature remains neutral with regard to jurisdictional claims in published maps and institutional affiliations.

\section{Author details}

${ }^{1}$ Department of Physiotherapy, Federal University of São Carlos, Rod. Washington Luís, km 235, São Carlos, São Paulo 13565-905, Brazil.

${ }^{2}$ Department of Medicine, Federal University of São Carlos, Rod. Washington Luís, km 235, São Carlos, São Paulo 13565-905, Brazil. ²Department of Physical Therapy, College of Applied Health Sciences, University of Illinois at Chicago, Chicago, IL, USA. ${ }^{4}$ Cardiopulmonary Physiotherapy Laboratory, Department of Physiotherapy, Federal University of São Carlos, Rod. Washington Luís, km 235, São Carlos, São Paulo 13565-905, Brazil.

Received: 19 December 2017 Accepted: 6 August 2018

Published online: 31 October 2018

References

1. de Faria Santarém GC, Cleva RD, Santo MA, Bernhard AB, Gadducci AV, Greve JMDA, et al. Correlation between Body Composition and Walking Capacity in Severe Obesity. PloS one. 2015;10:e0130268.

2. Busetto L, Dixon J, Luca MD, Shikora S, Pories W, Angrisani L. Bariatric surgery in class I obesity. Obesity surgery. 2014;24:487-519.

3. Liu Z, Brooks RS, Ciappio ED, Kim SJ, Crott JW, Bennett G, et al. Diet-induced obesity elevates colonic TNF-a in mice and is accompanied by an activation of Wnt signaling: a mechanism for obesity-associated colorectal cancer. J Nutr Biochem. 2012:23:1207-13.

4. Lee H, Lee IS, Choue R. Obesity, inflammation and diet. Pediatric gastroenterology, hepatology \& nutrition. 2013;16:143-52.

5. Beriault K, Carpentier AC, Gagnon C, Ménard J, Baillargeon JP, Ardilouze JL, et al. Reproducibility of the 6-minute walk test in obese adults. International journal of sports medicine. 2009;30:725-7. 
6. Fandiño J, Benchimol AK, Coutinho WF, Appolinário JC. Cirurgia Bariátrica: aspectos clínico- cirúrgicos e psiquiátricos. R. Psiquiatr. 2004;26:47-51.

7. Rubino F, Schauer PR, Kaplan LM, Cummings DE. Metabolic surgery to treat type 2 diabetes: clinical outcomes and mechanisms of action. Annual review of medicine. 2010;61:393-411.

8. Balsiger BM, Murr MM, Poggio JL, Sarr MG. Bariatric Surgery. Surgery for weight control in patients morbid obesity. Med Clin Nort Am. 2000;84:477-89.

9. Vanhecke TE, Franklin BA, Miller WM, deJong AT, Coleman CJ, McCullough PA. Cardiorespiratory fitness and sedentary lifestyle in the morbidly obese. Clinical Cardiology. 2009;32:121-4

10. Filipovic A, Kleindöder H, Plück D, Hollmann W, Bloch W, Grau M. Electromyostimulation-a systematic review of the effects of different electromyostimulation methods on selected strength parameters in trained and elite athletes. J Strength Cond Res. 2012;26:2600-14.

11. Kemmler W, Bebenek M, Engelke K, Stengel SV. Impact of whole-body electromyostimulation on body composition in elderly women at risk for sarcopenia: The Training and ElectroStimulation Trial (TEST-III). 2014;36:395-406.

12. Sillen M, Franssen FME, Gosker HR, Wouters EFM, Spruit MA. Metabolic and Structural Changes in Lower-Limb Skeletal Muscle Following Neuromuscular Electrical Stimulation: A Systematic Review. PLoS ONE. 2013;8:9.

13. Kemmler W, von Stengel S. Whole-body electromyostimulation as a means to impact muscle mass and abdominal body fat in lean, sedentary, older female adults: Subanalysis of the TEST- III trial. Clinical Interventions in Aging. 2013:8:1353-64.

14. Arija-Blázquez A, Ceruelo-Abajo S, Díaz-Merino MS, Godino-Durán JA, MartínezDhier L, Martin JLR, et al. Effects of electromyostimulation on muscle and bone in men with acute traumatic spinal cord injury: A randomized clinical trial. The journal of spinal cord medicine. 2014;37:299-309.

15. Consenso Bariátrico Brasileiro: Sociedade Brarsileira de Cirurgia Bariátrica e Metabólica http://www.saudedireta.com.br/docsupload/1425665481 consenso_ bariatrico.pdf.

16. Castello V, Simões RP, Bassi D, Catai AM, Arena R, Borghi-Silva A. Impact of aerobic exercise training on heart rate variability and functional capacity in obese women after gastric bypass surgery. Obesity Surgery. 2011;21:1739-49.

17. Ekman MJ, Klintenberg M, Björck U, Norström F, Ridderstrale M. Six-minute walk test before and after a weight reduction program in obese subjects. Obesity, 2013;21E236-E243.

18. Baecke JA, Burema J. Frijters JE. A short questionnaire for the measurement of habitual physical activity in epidemiological studies. Am J Clin Nutr. 1982;36:936-42.

19. Borg GAV. Psychophysical bases of perceived exertion. Medicine and science in sports and exercise. 1982;14:377-81.

20. ATS Committee on Proficiency Standards for Clinical Pulmonary Function Laboratories. ATS statement: guidelines for the six-minute walk test. Am J Respir Crit Care Med. 2002;166:111-117.

21. Gutch M, Kumar S, Razi SM, Gupta KK, Gupta A. Assessment of insulin sensitivity/ resistance. Indian journal of endocrinology and metabolism. 2015;19:160-4.

22. Bouchard DR, Janssen I. Dynapenic-obesity and physical function in older adults. The journals of gerontology. Series A, Biological sciences and medical sciences. 2010;65:71-7.

23. Colley D, Cines B, Current N, Schulman C, Bernstein S, Courville AB, et al, Assessing body fatness in obese adolescents: Alternative methods to Dual-Energy X-Ray Absorptiometry. The Digest. 2015;50:1-7.

24. Biospace. InBody 720 the precision body composition analyser: user's manual. Seoul: Biospace Co., Ltda. 2004.

25. Ling CH, de Craen AJ, Slagboom PE, Gunn DA, Stokkel MP, Westendorp RG, et al. Accuracy of direct segmental multi-frequency bioimpedance analysis in the assessment of total body and segmental body composition in middle-aged adult population. Clin Nutr. 2011;30:610-5.

26. Gobbo LA, Cyrino ES, Petroski EL, Cardoso JR, Carvalho FO, Romanzini M, et al. Validação de equações antropométricas para a estimativa da massa muscular por meio de absortometria radiológica de dupla energia em universitários do sexo masculino. Rev Bras Med Esporte. 2008;14:376-80.

27. Marcus RL, Addison O, Dibble LE, Foreman KB, Morrell G, LaStayo P. Intramuscular adipose tissue, sarcopenia, and mobility function in older individuals. J Aging Res. 2012. https://doi.org/10.1155/2012/629637.

28. Borghi-Silva A, Di Thommazo L, Pantoni CB, Mendes RG. Salvini TdeF, Costa D. Non- invasive ventilation improves peripheral oxygen saturation and reduces fatigability of quadriceps in patients with COPD. Respirology. 2009;14:537-44.

29. Miha Bodytec. http://www.miha-bodytec.com/pt/pagina-inicial/. Acessed 21 Jan 2015.
30. Kemmler W, Engelke K, Lauber D, Weineck J, Hensen J, Kalender WA. Impact of intense exercise on physical fitness, quality of life, and bone mineral density in early postmenopausal women. Year 2 results of the Erlangen Fitness Osteoporosis Prevention Study (EFOPS). Arch Int Med. 2004;164:1084-91.

31. Kemmler W, Engelke K, von Stengel S, Lauber D, Beeskow C, Pintag R, Weineck J, Kalender WA. Long term exercise favorably affects menopausal risk factors: The EFOPS-study. J Strength Cond Res. 2007;21:232-9.

32. Kemmler W, Lauber D, Von Stengel S, Engelke K. Developing maximum strength in older adults-A series of studies. In: Gießing J, Fröhlich M, Preuss P, editors. Current Results of Strength Training Research. Göttingen: Cuvillier Verlag; 2005. p. 114-33.

33. Kemmler W, Schliffka R, Mayhew JL, von Stengel S. Effects of wholebody electromyostimulation on resting metabolic rate, body composition, and maximum strength in postmenopausal women: the Training and ElectroStimulation Trial. Journal of strength and conditioning research / National Strength \& Conditioning Association. 2010;24:1880-1887

34. Cohen J. Statistical power analysis for the behavioral sciences. 2nd. edition. Hillsdale, NJ: Lawrence Erlbaum Associates; 1988.

35. Rowell B, O'Leary DS. Reflex control of the circulation during exercise: chemoreflexes and mechanoreflexes. J Appl Physiol. 1990;69:407-18.

36. Jones KB. Experience with the Roux-en-Y gastric bypass and commentary on current trends. Obes Surg. 2000;10:183-5.

37. Zilbertein B, Neto MG, Ramos AC, Cardoso A. O papel da cirurgia no tratamento da obesidade. Rev Bras Med. 2002;59:258-64.

38. Alam I, Lewis MJ, Lewis KE, et al. Influence of bariatric surgery on indices of cardiac autonomic control. Autonomic neuroscience : basic \& clinical. 2009; 151:168-17.

39. Maser R, Lenhard J, Irgau I, Wynn GM. Impact of Surgically Induced Weight Loss on Cardiovascular Autonomic Function: One-year Follow-up. Obesity. 2007:15:364-9.

40. Nault I, Nadreau E, Paquet C, Brassard P, Marceau P, Biron S, et al. Impact of bariatric surgery- induced weight loss on heart rate variability. Metabolism, clinical and experimental. 2007;56:1425-30.

41. Coen PM, Tanner CJ, Helbling NL, Dubis GS, Hames KC, Xie H, et al. Clinical trial demonstrates exercise following bariatric surgery improves insulin sensitivity. J Clin Invest. 2015;248-257(2015):125.

42. Wiklund $M$, Sundqvist $E$, Fagevik MO. Physical Activity in the Immediate Postoperative Phase in Patients Undergoing Roux-en-Y Gastric Bypass-a Randomized Controlled Trial. Obesity surgery. 2015;25:2245-50.

43. Borghi-Silva A, Mendes RG, Trimer R, Junior GC, et al. Current trends in reducing cardiovascular disease risk factors from around the world: Focus on cardiac rehabilitation in Brazil. Progress in Cardiovascular Diseases. 2014:56:536-42.

44. Cipriano GJ, Neves LMT, Cipriano GFB, Chiappa GR, Borghi-Silva A. Cardiovascular disease prevention and implications for worksite health promotion programs in Brazil. Progress in Cardiovascular Diseases. 2014:56:493-500.

45. Evans RK. The Role of Physical Activity Participation in Weight Loss Outcomes Following Weight Loss Surgery. American Journal of Lifestyle Medicine. 2010;4:124-9.

46. Fernhall B, Borghi-Silva A, Babu AS. The Future of Physical Activity Research: Funding. Opportunities and Challenges. Progress in Cardiovascular Diseases. 2015;57:299-305.

47. Giusti V, Theytaz F, Di Vetta V, Clarisse M, Suter M, Taapy L. Energy and macronutrient intake after gastric bypass for morbid obesity: a 3-y observational study focused on protein consumption. Am J Clin Nutr. 2016;103:18-24.

48. Ito MK, Gonçalves VSS, Faria SLCM, Moizé V, Porporatti AL, Guerra ENS, et al. Effect of protein intake on the protein status and lean mass of post-bariatric surgery patients: a systematic review. Obes Surg. 2017;27: 502-12 Kittelson AJ, Stackhouse SK, Stevens-Lapsley JE. Neuromuscular Electrical Stimulation after total joint arthroplasty: A critical review of recent controlled studies. European Journal of Physical and Rehabilitation Medicine. 2013;49:909-920.

49. Neder JA, Sword D, Ward SA, Mackay E, Cochrane LM, Clark CJ. Home based neuromuscular electrical stimulation as a new rehabilitative strategy for severely disabled patients with chronic obstructive pulmonary disease (COPD). Thorax. 2002;57:333-7.

50. Vivodtzev I, Pépin $J$, Vottero G, Mayer $V$, Porsin B, Lévy $P$, et al. Improvement in quadriceps strenght and dyspnea in daily tasks after 1 
month of electrical stimulation in severely deconditioned and malnourished COPD. Chest. 2006;129:1540-8.

51. Kondo T, Ono K, Kitano S, Matsuyama R, Goto R, Suico MA, et al. Mild electrical stimulation with heat shock reduces visceral adiposity and

improves metabolic abnormalities in subjects with metabolic syndrome or type 2 diabetes: Randomized crossover trials. EBioMedicine. 2014;1:80-9.

Ready to submit your research? Choose BMC and benefit from:

- fast, convenient online submission

- thorough peer review by experienced researchers in your field

- rapid publication on acceptance

- support for research data, including large and complex data types

- gold Open Access which fosters wider collaboration and increased citations

- maximum visibility for your research: over $100 \mathrm{M}$ website views per year

At $\mathrm{BMC}$, research is always in progress.

Learn more biomedcentral.com/submissions 